\title{
Tunable Narrowband Tm:YAP Laser Using a Transversally Chirped Volume Bragg Grating
}

\author{
Quentin Berthomé $^{1,2}$, Arnaud Grisard ${ }^{3}$, Basile Faure ${ }^{1}$, Grégoire Souhaité ${ }^{1}$, Eric Lallier ${ }^{3}$, Antoine Godard $^{2}$, \\ Vadim Smirnov ${ }^{4}$, Ruslan Vasilyeu ${ }^{4}$ \\ 1. Teem Photonics, 61 Chemin du Vieux Chêne, Innovallée, 38240 Meylan, France \\ 2. ONERA, DPHY, Université Paris Saclay, F-91123 Palaiseau, France \\ 3. Thales Research \& Technology, 1 Avenue Augustin Fresnel, 91767 Palaiseau, France \\ 4. OptiGrate Corp (an IPG Photonics Company), 562 South Econ Circle, Oviedo, Florida 32765, USA
}

Tunable long wave infrared (LWIR) coherent sources emitting between 8 and $12 \mu \mathrm{m}$ can be used for versatile remote gas sensing. In ref[1] we implemented a parametric oscillator in which wavelength tunability was achieved by temperature tuning of the crystal indices of refraction. It is well known that a similar wavelength range can be addressed by tuning the pump wavelength rather than the crystal temperature [2]. In addition, pump tuning can be significantly faster than temperature tuning. In this context we also need a narrow band spectrum to control the spectral output of the optical parametric oscillator, which is based on Vernier spectral filtering in doubly-resonant cavities. Seger and his team exhibited single longitudinal mode operation in a microlaser tuned with transversally chirped volume Bragg grating (TC-VBG) near $1 \mu \mathrm{m}$ [3]. We show how this concept can be applied to a different laser emitting at $2 \mu \mathrm{m}$.

We designed a Tm:YAP laser with a TC-VBG as output coupler. The cavity, shown in Fig. 1 (a), is composed of a high reflective dielectric concave mirror around $2 \mu \mathrm{m}$, a $4 \mathrm{~mm}$ long $4 \%$ at. thulium doped a-cut YAP rod and a TC-VBG with 1940 to $1960 \mathrm{~nm}$ tuning range, $85 \%$ reflectivity and $0.8 \mathrm{~nm}$ bandwidth. We pump the cavity with a $9 \mathrm{~W}$ laser diode emitting near $790 \mathrm{~nm}$. Depending on the output wavelength, this laser has a threshold between $1.8 \mathrm{~W}$ and $2.1 \mathrm{~W}$ and 14 to $17 \%$ of efficiency. With this cavity we obtained a continuous wave laser emitting up to five cavity modes witch gives a $0.2 \mathrm{~nm}$ wide spectrum with up to $1.5 \mathrm{~W}$ laser output power at $1948 \mathrm{~nm}$ under $7 \mathrm{~W}$ pumping. Working at low power above the threshold reduces the laser spectral width below $0.1 \mathrm{~nm}$ but it quickly reaches $0.2 \mathrm{~nm}$ wide (see Fig 1 (b)). We plan to push on to obtain narrower linewidth.

(a)
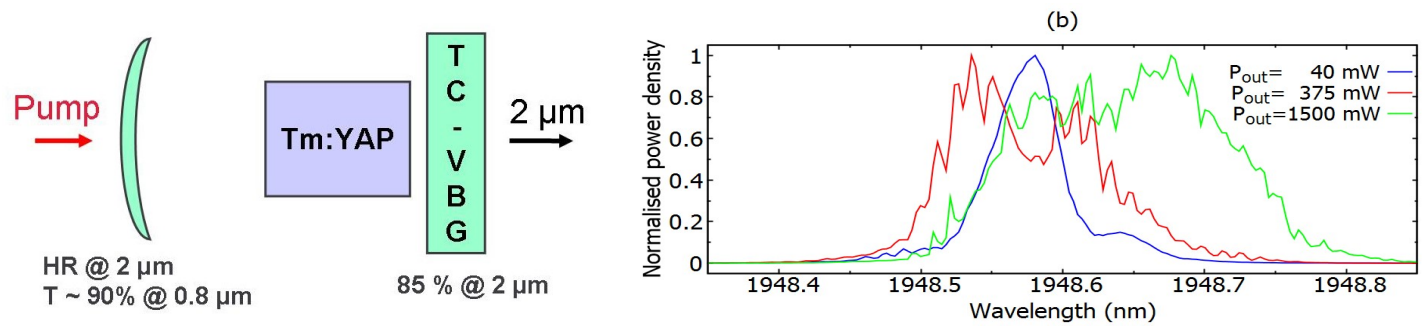

Fig. 1 (a) Schematic of the laser cavity with the TCVBG (b) Laser spectrum at $1948.6 \mathrm{~nm}$ obtained with an optical spectrum analyser with $0.050 \mathrm{~nm}$ resolution for different output powers.

With transversal translation of the TC-VBG over its $20 \mathrm{~mm}$ length, the wavelength is tuned continuously from $1940 \mathrm{~nm}$ to $1960 \mathrm{~nm}$. After parametric downconversion to the LWIR, this range would translate to $\sim 500$ nm near $10 \mu \mathrm{m}$, which would be enough to acquire the same spectrum as presented in ref [1]. This is to our knowledge, the widest tuning range near $2 \mu \mathrm{m}$ obtained with this method. This leads to an efficient, simple, compact, tunable diode pumped solid state laser with narrow linewidth near $2 \mu \mathrm{m}$. There is no theoretical limitation to adapt this solution over the entire thulium gain band. However, practical limitations to TC-VBG chirp rate and size limit the total tuning range accessible with one TC-VBG. The next step will be to make the laser work in pulsed regime with either passive [4] or active Q-switching.

\section{Example References}

[1] Q. Clément, J.-M. Melkonian, J.-B. Dherbecourt, M. Raybaut, A. Grisard, E. Lallier, B. Gérard, B. Faure, G. Souhaité, and A. Godard, "Longwave infrared, single-frequency, tunable, pulsed optical parametric oscillator based on orientation-patterned GaAs for gas sensing", Opt. Lett. 40, $2676(2015)$

[2] F. Gutty, A. Grisard, C. Larat, D. Papillon, M. Schwartz, B. Gérard, R. Ostendorf, M. Rattunde, J. Wagner, and E. Lallier, "140W Peak Power Laser System Tunable in the LWIR", Opt. Expr. 25, 18897 (2017)

[3] K. Seger, N. Meiser, C. Canalias, V. Pasiskevicius, and F. Laurell, "Tunable, passively Q-switched single-longitudinal-mode Nd:YVO 4 laser using a chirped volume Bragg grating", Appl. Phys. B 109, 99-103 (2012)

[4] A. Grisard, B. Faure, G. Souhaité and E. Lallier, "High energy single frequency passively Q-switched 2-micron microlaser in thuliumdoped yttrium aluminium perovskite", paper ATu2A.39 in ASSL, OSA (2014) 